\title{
Intussusception in intestinal lymphoma: the role of colonoscopy
}

\section{Eran Goldin and Eugene Libson}

Department of Gastroenterology and Radiology, Hadassah University Hospital, Hebrew University-Hadassah Medical School, Jerusalem, Israel

\begin{abstract}
Summary: Intussusception is a well known complication and sometimes the presenting symptom of intestinal lymphoma. The final diagnosis in most patients is made by surgery.

We report a 12 year old boy with intussusception in whom the diagnosis was established by colonoscopy and surgery was avoided.
\end{abstract}

\section{Introduction}

Intussusception is the most frequently recognized cause of intestinal obstruction in young children, in over $90 \%$ of cases the cause remaining unclear. In older children and in adults most of the intussusceptions are caused by malignant tumours, primary intestinal neoplasms or metastatic involvement of the bowel. In children over the age of 6 most of these tumours are primary intestinal lymphoma (Wayne et al., 1976).

The ileocaecal area is the most frequent site of intussusception. In most series the diagnosis of intussusception and its underlying cause was rarely made preoperatively and surgical intervention was necessary almost every time (Wayne et al., 1976; Karakousis et al., 1974). In the case herein reported the diagnosis of the aetiology of the intussusception was made by colonoscopy and surgery was therefore avoided.

\section{Case report}

A 12 year old boy enjoyed good health until a month before admission. At this time he began to suffer from abdominal pain and later he had bloody bowel movements. On admission physical examination revealed a right lower quadrant abdominal mass. A barium enema (Figure 1) demonstrated the presence of an intussusception in the ileocaecal area which was irreducible by barium pressure.

Correspondence: E. Goldin, M.D., Department of Gastroenterology, Hadassah University Hospital, P.O. Box 12000, Jerusalem, Israel

Accepted: 9 July 1986

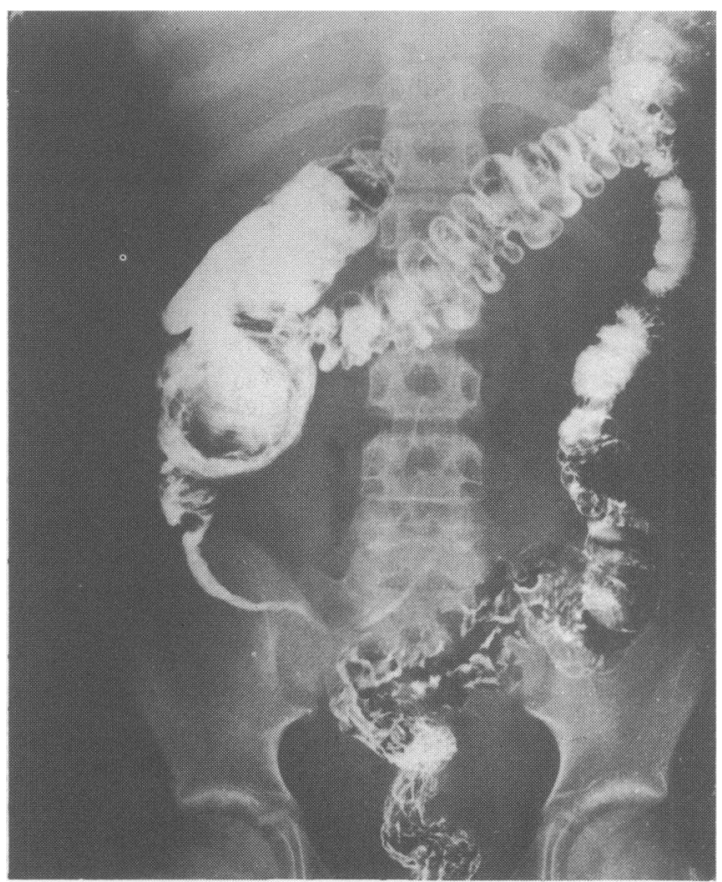

Figure 1 Barium enema showing intussusception in the ileocaecal area.

A colonoscopy was performed (Figure 2) and a big oval and quite soft mass that 'filled' almost the entire caecum was found. Biopsies were obtained and a diagnosis of Burkitt's lymphoma was established. Abdominal computed tomographic scan disclosed

(C) The Fellowship of Postgraduate Medicine, 1986 


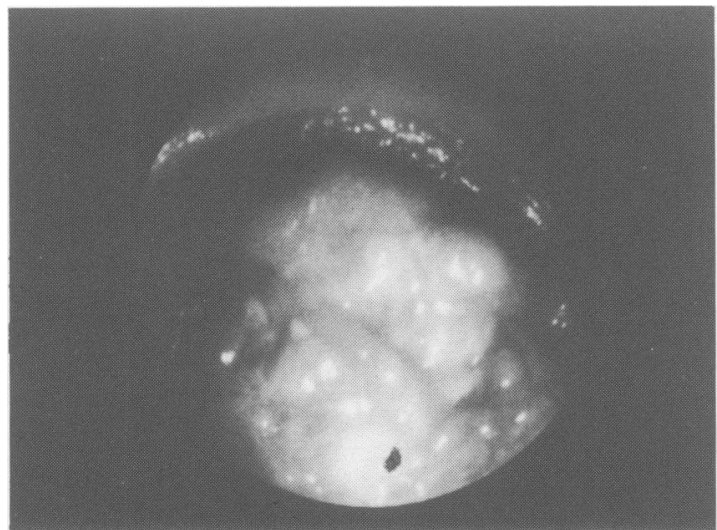

Figure 2 Colonoscopy. A big mass is seen in the caecum.

extension of the tumour into the mesenteric lymph nodes and the retroperitoneum.

The patient was treated with chemotherapy, and a slow improvement was obtained. The obstructive symptoms disappeared and his general condition improved.

\section{References}

BENSON, C.D., HOYA, J.R. \& FISHER, H. (1963). Intussusception in infants and children. Archives of Surgery, 86, 745.

KARAKOUSIS, C., HOLYOKE, E.D. \& DOUGLASS, H.D. (1974). Intussusception as a complication of malignant neoplasm. Archives of Surgery, 109, 515.

MESTEL, A.L. (1959). Lymphosarcoma of the small intestine

\section{Comments}

In over $90 \%$ of young children with intussusception the cause remains obscure (Benson et al., 1963). Older children and adults develop intussusception less often and in more than $50 \%$ of the patients the underlying cause is malignant disease. In the older childhood group with this condition the diagnosis is usually ileal lymphoma until proven otherwise (Wayne et al., 1976; Mestel, 1959). In adults, melanoma, adenocarcinoma of the colon and metastatic tumours may also present as an intussusception (Karakousis et al., 1974). In spite of the fact that chemotherapy is the treatment of choice for lymphoma, in most of the reports a diagnostic laparotomy is recommended.

Colonoscopy is a low risk approach to recognize and to make a histological diagnosis of the pathological process before treatment. In adults, colonoscopy may prevent surgery or may give the surgeon fuller information in cases of resectable tumour. In most older children with lymphoma, surgery is not necessary. In this group, we think that colonoscopy must be the preferred technique in cases where the process is within the colon or the terminal ileum and a histological diagnosis is mandatory. Surgery must be reserved for those cases with complete obstruction or where the intussusception is high in the small bowel.

in infancy and childhood. Annals of Surgery, 149, 87.

WAYNE, E.R., CAMPBELL, J.B., KOSLOSKE, A.M. \& BURR INGTON, J.D. (1976). Intussusception in the older child suspect lymphosarcoma. Journal of Pediatric Surgery, 5, 789. 